\title{
Side-Chain Fragmentation of Alkylated \\ Cysteine Residues in Electron Capture Dissociation Mass Spectrometry
}

\author{
R. J. Chalkley, C. S. Brinkworth, and A. L. Burlingame \\ Mass Spectrometry Faculty, University of California San Francisco, San Francisco, California, USA
}

\begin{abstract}
The recent development of novel fragmentation processes based on either electron capture directly or transfer from an anion show great potential for solving problems in proteomics that are intractable by the more widely employed thermal-based fragmentation processes such as collision induced dissociation. The dominant fragmentation occurring upon electron capture dissociation of peptides is cleavage of $\mathrm{N}-\mathrm{C} \alpha$ bonds in the peptide backbone to form $\mathrm{c}$ and $\mathrm{z}$. ions. In the case of disulfide-linked peptides, it has also been shown that electron capture on one of the cystine sulfur atoms is favored, resulting in cleavage of the disulfide bond. In this study, we report that electron capture on the sulfur of alkylated cysteine residues is also a dominant process, causing cysteine side-chain loss from $z$. ions. (J Am Soc Mass Spectrom 2006, 17, 1271-1274) (C) 2006 American Society for Mass Spectrometry
\end{abstract}

$\mathrm{T}$ The development of two novel energy deposition processes in mass spectrometry, electron capture dissociation (ECD) [1] and electron-transfer dissociation (ETD) [2], are expected to have a significant impact within the fields of biomacromolecular analysis and proteomics in particular. These two types of radicalbased fragmentation processes involve cleavage of bonds adjacent to sites of electron capture. Unlike other fragmentation processes, such as collision induced dissociation (CID), the bonds that are cleaved are not the most labile within the molecule, and the cleavages observed are less dependent on the peptide sequence, leading to cleavages between most amino acids in the peptide backbone somewhat independent of molecular size. ECD has already been demonstrated to be useful for the analysis of labile post-translational modifications that are refractory to analysis by CID such as phosphorylation $[3,4]$ and O-glycosylation $[5,6]$. It has also been proven to be a powerful tool for fragmentation analysis of intact proteins [7-9].

The dominant fragmentation in ECD and ETD of peptides is the formation of $\mathrm{c}$ and $\mathrm{z}$ - ions. It has also been shown that preferential capture in the disulfide bridge of disulfide-linked peptides occurs with cleavage of the linking bond to form a neutral sulfhydryl, $\mathrm{R}-\mathrm{SH}$, and a thiyl radical species, S-R [10].

The primary approach for peptide and protein identification in proteomics studies is to digest a protein with an endoproteolytic enzyme, most commonly tryp-

Published online June 30, 2006

Address reprint requests to Dr. R. J. Chalkley, Mass Spectrometry Facility, University of California San Francisco, 521 Parnassus Ave., Room C-18, San Francisco, CA 94143-0446, USA. E-mail: chalkley@cgl.ucsf.edu sin, and then analyze peptides. To improve the efficiency of digestion and analysis, it is a standard strategy to reduce disulfide bonds and alkylate the resulting free sulfhydryls to prevent bridge re-formation. This breaks the protein's tertiary structure and allows trypsin better access to the whole protein sequence.

The behavior of peptides containing alkylated cysteine residues in ECD has not been reported. Here we show that under ECD conditions, peptides containing cysteine residues modified with a variety of common alkylating agents form dominant fragment ions where the modified cysteine side chain is lost to form $z^{\prime}$ type ions.

\section{Methods}

The tryptic digest of a six protein mixture of bovine serum albumin, human transferrin, alcohol dehydrogenase, cytochrome $c, \beta$-galactosidase, and lysozyme $c$ was purchased from Dionex (Sunnyvale, CA). In this mixture, cysteines are carboxymethylated. Human TRAF2, where the cysteines were carbamidomethylated and bovine fetuin, where cysteines were pyridylethylated, were both digested in-house. All data were acquired on a commercial LTQ-FT (Thermo, San Jose, CA) fitted with an electron gun. Samples were analyzed by LC-MS using an Eksigent 1D NanoLC system (Dublin, CA). The column was a 100 um ID x $150 \mathrm{~mm}$ selfpacked column with Ultro120 (Peeke Scientific, Redwood City, CA) C-18 resin. The flow rate was 350 $\mathrm{nl} / \mathrm{min}$, solvents were water $/ 0.1 \%$ formic acid $(\mathrm{A})$ and acetonitrile $/ 0.1 \%$ formic acid (B), and the gradient was $5-40 \%$ B over $30 \mathrm{~min}$. All components were selected for 


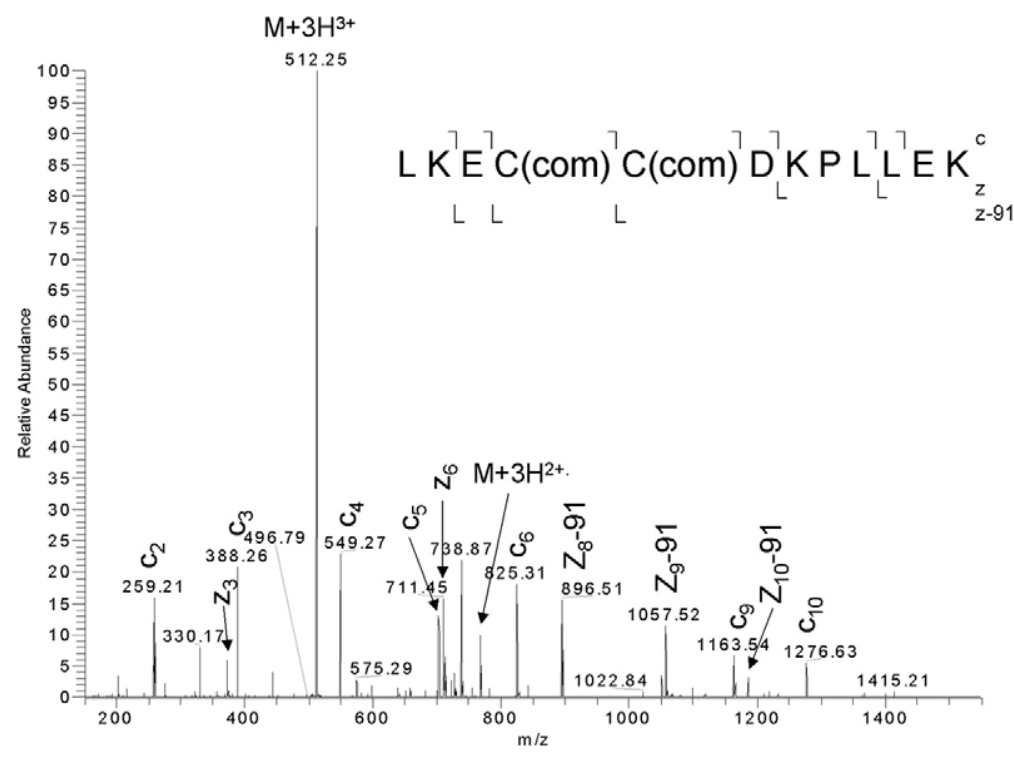

Figure 1. ECD spectrum of peptide where cysteines have been carboxymethylated (com).

ECD fragmentation in a data-dependent fashion. For ECD fragmentation automatic gain control was set to 1 $\times 10^{5}$ ions and spectra were the sum of two microscans.

\section{Results}

A protein standard digest mixture, where cysteine residues were carboxymethylated, was analyzed by LC-MS with automated selection of observed precursor ions for ECD fragmentation analysis. Figure 1 shows the annotated ECD mass spectrum of an $[\mathrm{M}+3 \mathrm{H}]^{3+}$ ion at $m / z 512.254$ that corresponds to the peptide LKECCDKPLLEK from bovine serum albumin. An extensive $\mathrm{c}$ ion series is observed, but only two $\mathrm{z}$ - ions are present $\left(z_{3}\right.$ and $\left.z_{6}\right)$. However, there are a series of intense ions that are $91 \mathrm{Da}$ lower in mass than expected $\mathrm{z} \cdot$ ions, and these only appear associated with fragment ions that correspond to parts of the peptide that contained one or two carboxymethylated cysteine residues. This observation suggests that these fragments are due to a loss from the carboxymethylated cysteine to form a modified residue with a mass of $70 \mathrm{Da}$. Simply losing the side chain of a carboxymethylated cysteine residue would correspond to a loss of 92 Da creating a residue of mass of $69 \mathrm{Da}$, not $70 \mathrm{Da}$. However, it has been observed in ECD that as well as the usually reported $c$ and $z \cdot$ ions, sometimes $c \cdot$ and $z^{\prime}$ (or $c-1$ and $z+1$ ) ions can be detected. The fragment observed is therefore a $z^{\prime}$ ion where the cysteine side chain has been eliminated with the abstraction of a hydrogen.

To ascertain whether this phenomenon was unique to carboxymethylated cysteine residues, a peptide digest where cysteines were carbamidomethylated was analyzed. Figure 2 shows the ECD spectrum of $[\mathrm{M}+3 \mathrm{H}]^{3+} m / z 555.633$ corresponding to a peptide KTATFENIVCVLNR. In this spectrum, a complete c ion series was observed together with several $z \cdot$ ions.
However, although a few $\mathrm{z}$ - ions including the peptide sequence containing the carbamidomethylated cysteine were observed, a much more intense series of fragment ions were present that corresponded to species $90 \mathrm{Da}$ smaller in mass than the predicted $\mathrm{z}$. ion series. The side chain of a carbamidomethylated cysteine residue corresponds to $91 \mathrm{Da}$, so again these ions correspond to a series of $z^{\prime}$ ions formed after loss of the cysteine side chain and hydrogen abstraction.

Further, a tryptic digest of bovine fetuin, where reduced cysteines had been pyridylethylated, was also analyzed, and similar fragment ions were observed from modified-cysteine containing peptides with the apparent formation of a residue of mass $70 \mathrm{Da}$ (data not shown).

\section{Discussion}

Radical neutral loss of the amino acid side chains in ECD from the $z$. fragment ions, including those from cysteine $(\cdot \mathrm{SH})$, have recently been described [11]. Here we describe a similar fragmentation process observed in ECD mass spectra of peptides containing alkylated cysteine moieties. This ion type corresponds to $\mathrm{z}^{\prime}$ ions, where the modified cysteine side chain has been eliminated. Importantly, conventional $\mathrm{z} \cdot$ ions are only sometimes also observed and, if so, are lower in intensity than those formed after alkylated cysteine side chain loss. It is interesting that $\mathrm{z}^{\prime}$ type ions are observed for these elimination product ions whereas $z \cdot$ ions are seen for fragments containing the alkylated cysteine side chain. Another important observation (or lack of observation) is that this eliminated ion type is only observed from $\mathrm{z}$ ions; never from $\mathrm{c}$ ions. This suggests the process of formation of this ion type is due to the free radical state of the $z \cdot$ ion. The fact that this ion type is observed for a whole series of $\mathrm{z}$ ions supports the theory that the 


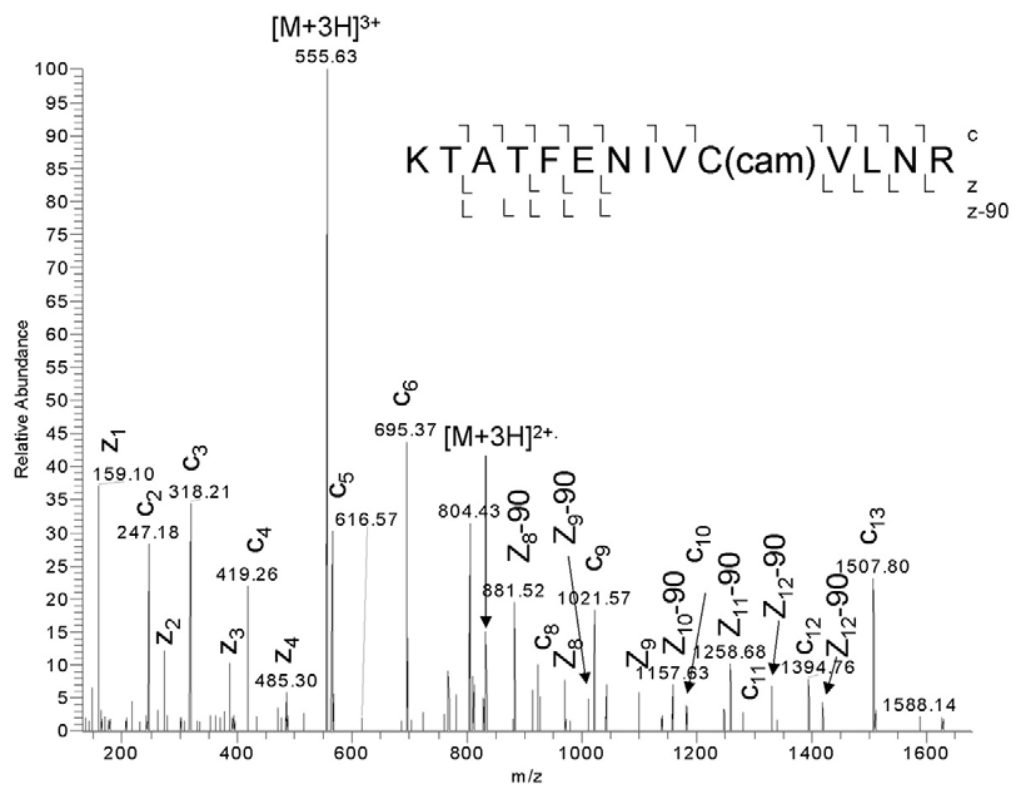

Figure 2. ECD spectrum of a peptide in which the cysteine is carbamidomethylated (cam).

ECD fragmentation processes can drive free radical reaction cascades to cause fragmentation remote to the site of electron capture [11, 12]. In our studies in spectra of peptides containing two alkylated cysteine residues, only the loss of one side chain is observed. As the $z^{\prime}$ ion is an even electron species, whereas the $z \cdot$ ion is a free radical, we propose that the cysteine side chain is being lost as a free radical to leave a stable even electron species and, hence, a second cysteine side chain cannot be lost by the same process.
Theoretical calculations indicate that when the amino acid in question is in the $\mathrm{N}$-terminal position, the radical localized upon the $\alpha$-carbon (1) initiates the loss [11] (Scheme 1a). However, radical loss of the amino acid side chain can also occur even when the residue is not in the N-terminal position as a result of 1,5-hydrogen shifts that propagate the radical along the backbone to any of the $\alpha$-carbon positions (Scheme $1 \mathbf{b}$ ). Calculations for both of these scenarios indicate that these processes are exothermic and require only moderate activation energy. In

(a)<smiles>[2H]N(C([14CH3])=O)N([18OH])C(=O)C=C</smiles>

(b)

(1)

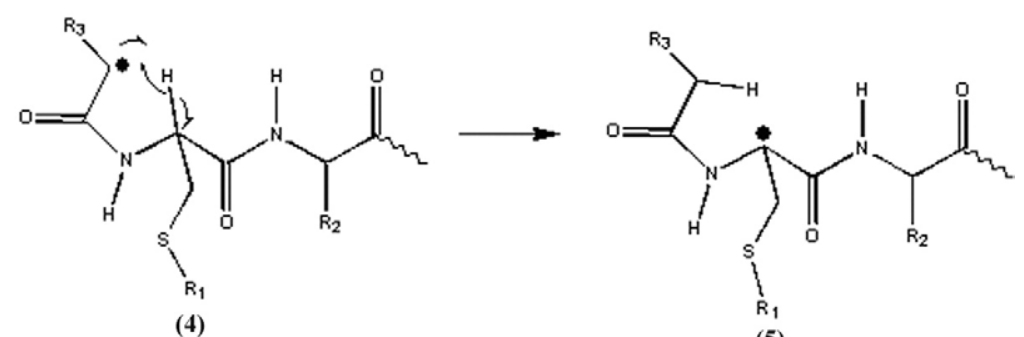

(4)
(2)

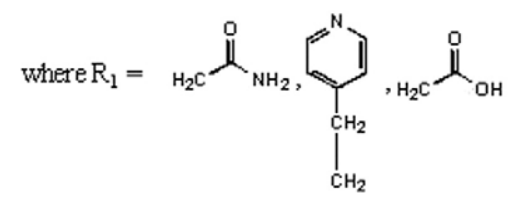


addition, the size of sulfur's electron cloud compared with oxygen and carbon means that it can better stabilize the additional electron density. This should result in lower heats of formation of these types of radicals favoring their formation.

We propose the following to explain the fragmentation observed in Figures 1 and 2. Following dissociation of the peptide backbone and formation of the $z$. fragment ion (1), the radical can initiate one of three scenarios. First, it can remain on the N-terminal $\alpha$ carbon resulting in the standard $z$. fragment ion. Second, the radical can trigger cleavage of the $\beta-\gamma$ bond, liberating the $\mathrm{N}$-terminal amino acid side chain as the neutral radical (3) and generating the $\mathrm{z}^{\prime}$ fragment ion (2) (Scheme 1a). This type of process generated the large even-electron z8-91 and z9-91 fragment ions in preference to the standard $z$. fragment ion (1) observed in Figure 1 . The liberated $\cdot \mathrm{SCH} 2 \mathrm{COOH}$ radical is more stable than the corresponding $\mathrm{z}$ - fragment ion as a result of both the resonance stabilization and the size of electron cloud of sulfur. Or third, the radical can be propagated along the backbone (Scheme $\mathbf{1 b}$ ), neutral radical loss of the cysteine side chain will be favored because of sulfur's ability to better stabilize the extra electron as compared to oxygen and carbon present in the other amino acids, i.e., (5) in Scheme $\mathbf{1 b}$ is similar in structure to (1) in Scheme 1a and can lose the side chain resulting in an even electron $z^{\prime}$ fragment ion (2). This is observed in Figure 1 as indicated by z10-91. Notice that there is no z10. fragment observed suggesting that the cleavage of both the $\mathrm{NH}-\alpha \mathrm{C}$ and $\beta-\gamma$ bond occurs in a concerted process rather than stepwise. In Figure 2, both the $z$. and z-90 fragments are present. The leaving group in this system is the $\mathrm{SCH} 2 \mathrm{C}(\mathrm{O}) \mathrm{NH} 2$ radical, which cannot resonance stabilize the radical. Therefore, it would be expected that the energetics of this process must be closer to that of the heat of formation of the $\mathrm{z}$. fragment ion allowing both fragment ions to be observed.

\section{Conclusions}

During the coming years, the use of ECD (and ETD) for analysis of proteins and peptides is expected to become increasingly widespread and common. Hence, it is important to understand these mechanisms of fragmentation so that it is possible to predict the fragment ions observed and data can be analyzed comprehensively by database search engines. This work shows that for analysis of alkylated-cysteine containing peptides, ions formed by the side-chain loss from the cysteine residue will be dominant C-terminally derived ions in the ECD (and presumably ETD) spectra and the corresponding $\mathrm{z}$ ions may not even be observed. Database search engines, therefore, need to be adapted to take this into consideration.

\section{Acknowledgments}

The authors thank Kati Medzihradzsky, David Maltby, and Shenheng Guan for useful discussions of these results. This work was supported by NIH NCRR grants RR01614 and RR019934.

\section{References}

1. Zubarev, R. A. Electron-capture dissociation tandem mass spectrometry. Curr. Opin. Biotechnol. 2004, 15, 12-16.

2. Coon, J. J.; Shabanowitz, J.; Hunt, D. F.; Syka, J. E. Electron transfer dissociation of peptide anions. J. Am. Soc. Mass Spectrom. 2005, 16, $880-882$.

3. Kjeldsen, F.; Haselmann, K. F.; Budnik, B. A.; Sorensen, E. S.; Zubarev, R. A. Complete characterization of posttranslational modification sites in the bovine milk protein PP3 by tandem mass spectrometry with electron capture dissociation as the last stage. Anal. Chem. 2003, 75, 2355-2361.

4. Shi, S. D.; Hemling, M. E.; Carr, S. A.; Horn, D. M.; Lindh, I.; McLafferty, F. W. Phosphopeptide/phosphoprotein mapping by electron capture dissociation mass spectrometry. Anal. Chem. 2001, 73, 19-22.

5. Mirgorodskaya, E.; Roepstorff, P.; Zubarev, R. A. Localization of O-glycosylation sites in peptides by electron capture dissociation in a Fourier transform mass spectrometer. Anal. Chem. 1999, 71, 4431-4436.

6. Vosseller K., Trinidad, J. C., Chalkley, R. J., Specht, C. G., Thalhammer, A., Lynn, A. J., Snedecor, J. H., Guan, S., Medzhiradszky, K. F., Maltby, D. A., Schoepfer, R., Burlingame, A. L. O-GlcNAc proteomics of postsynaptic density preparations using lectin weak affinity chromatography (LWAC) and mass spectrometry. Mol. Cell Proteom.2006,E-pub Feb 1, 2006.

7. Zubarev, R. A.; Horn, D. M.; Fridriksson, E. K.; Kelleher, N. L.; Kruger, N. A.; Lewis, M. A.; Carpenter, B. K.; McLafferty, F. W. Electron capture dissociation for structural characterization of multiply charged protein cations. Anal. Chem. 2000, 72, 563-573.

8. Pesavento, J. J.; Kim, Y. B.; Taylor, G. K.; Kelleher, N. L. Shotgun annotation of histone modifications: a new approach for streamlined characterization of proteins by top down mass spectrometry. J. Am. Chem. Soc. 2004, 126, 3386-3387.

9. Medzihradszky, K. F.; Zhang, X.; Chalkley, R. J.; Guan, S.; McFarland, M. A.; Chalmers, M. J.; Marshall, A. G.; Diaz, R. L.; Allis, C. D.; Burlingame, A. L. Characterization of tetrahymena histone H2B variants and post-translational populations by electron capture dissociation (ECD) Fourier transform ion cyclotron mass spectrometry (FT-ICR MS). Mol. Cell Proteom. 2004, 3, 872-886.

10. Zubarev, R. A.; Kruger, N. A.; Fridriksson, E. K.; Lewis, M. A.; Horn, D. M.; Carpenter, B. K.; McLafferty, F. W. Electron capture dissociation of gaseous multiply-charged proteins is favored at disulfide bonds and other sites of high hydrogen atom affinity. J. Am. Chem. Soc. 1999, 121, 2857-2862.

11. Fung, Y. M.; Chan, T. W. Experimental and theoretical investigations of the loss of amino acid side chains in electron capture dissociation of model peptides. J. Am. Soc. Mass Spectrom. 2005, 16, 1523-1535.

12. Leymarie, N.; Costello, C. E.; O'Connor, P. B. Electron capture dissociation initiates a free radical reaction cascade. J. Am. Chem. Soc. 2003, 125, 8949-8958. 